\title{
Para una reconstrucción genealógica y epistemológica del concepto de metacognición
}

\author{
For a genealogical and epistemological reconstruction of \\ the concept of metacognition
}

Juan Antonio González de Requena Farré .

Resumen

En las últimas décadas ha habido una creciente investigación acerca de la metacognición, que nos ha familiarizado con metatérminos como metaconocimiento o metaaprendizaje, entre otros. En este artículo intentamos reconstruir los modelos de la metacognición previos y los recientes paradigmas metacognitivos. También cuestionamos las implicaciones epistemológicas y las condiciones retóricas y socio-históricas de la acuñación y circulación del léxico metacognitivo.

Palabras clave: Metacognición, Paradigmas metacognitivos, Epistemología.

\section{Abstract}

In the last decades, there has been an increasing investigation on metacognition, which has made familiar to us meta-terms like metaknowledge or metalearning, among others. In this article, we try to reconstruct the previous models on metacognition and recent metacognitive paradigms. We also inquire epistemological implications and rethorical and socio-historical conditions of the coining and circulation of this metacognitive lexicon.

Key words: Metacognition, Metacognitive paradigms, Epistemology. 


\section{Introducción}

Ya a mediados de los ochenta, y ante la profusión de literatura referente a la metacognición, resultaba lícito preguntarse si estábamos asistiendo a la constitución de un nuevo paradigma en psicología. Los más críticos argumentaban que era tal la multiplicidad de significados proyectados sobre la metacognición que, quizás, este concepto estuviera sujeto a una cierta ambigüedad. La metacognición se asociaba no sólo al conocimiento reflexivo y consciente sobre los propios procesos cognoscitivos, sino también a la existencia de procesos de control relacionados con mecanismos autorregulatorios. Una multitud de sentidos paradigmáticos convergía en el léxico de la metacognición: conciencia reflexiva, teoría de la mente, procesamiento estratégico, autocontrol, autoeficacia, autoconcepto, representación de modelos mentales, recursividad, control ejecutivo y retroalimentación informativa, entre otros (Mayor, Suengas \& González, 1995). Ante esta abigarrada panoplia terminológica se planteó la sospecha de que nos encontrábamos ante un nuevo término de otra moda psicológica (Martí, 1995).

Actualmente, el término metacognición circula con normalidad en la investigación psicológica y se reproduce con total cotidianeidad en el discurso psicopedagógico y educativo. No obstante, en la reciente literatura sobre la metacognición se siguen observando los problemas de definición del término que, tan pronto designa el pensamiento sobre el pensamiento, como se refiere a la conciencia y/o regulación de la cognición. Por si fuera poco, actualmente se intenta asociar la metacognición a la epistemología personal (a las concepciones individuales sobre el conocimiento y el conocer) e, incluso, se introduce el término adicional de metacognición epistémica (las creencias sobre el conocer -teóricamente organizadas- que operan a nivel metacognitivo). En ese sentido, se postula que las creencias epistémicas (relativas a la fuente, estructura y justificación del conocimiento) son de carácter metacognitivo cuando implican el estar consciente de dichas creencias y la utilización de esas creencias sobre el conocimiento para regular la propia actividad cognitiva (Hofer \& Sinatra, 2010). 
En todo caso, no habría que asumir que un término en circulación profusa tiene un significado claro y distinto. Por eso, nos proponemos esclarecer epistemológicamente y explicitar genealógicamente las condiciones de posibilidad, las modalidades de conocimiento y las perspectivas cognitivas involucradas en la metacognición. Nuestra apuesta en este ensayo -y la vocación que nos guía- es doble. Por una parte, se introduce un tipo de reflexión epistemológica post-fundacionalista, que renuncia a cualquier pretensión de fundamentación apodíctica del conocimiento (a partir de algún tipo de autoevidencia cognitiva o metacognitiva). De ahí la importancia que tienen en nuestra investigación la semántica histórica y el análisis de la construcción social de los conceptos. Además, nuestra indagación se inscribe en la línea de una psicología crítica y constituye un intento de examinar los nexos entre la conceptualización psicológica y los intereses sociales, así como los trasfondos socio-históricos de construcción del conocimiento psicológico y los rendimientos ideológicos de los paradigmas hegemónicos en la autocomprensión disciplinar de la psicología (Parker, 2008).

La metacognición como retórica del "orden superior"

Los conceptos antecedidos con el prefijo meta tienen una venerable tradición en nuestro discurso filosófico y científico. El concepto metafísica marca la pauta para la construcción de estos metatérminos, aunque el concepto obtuviera su significado como consecuencia de un auténtico malentendido. Andrónico de Rodas clasificó como metafísicos aquellos escritos aristotélicos que venían después de la física; pero como en dichos escritos Aristóteles indagaba la posibilidad de una filosofía primera (esto es, una investigación fundamental de los primeros principios y causas de los seres que no se dejaría reducir a las ciencias específicas), la metafísica se terminó asociando, en la filosofía occidental, a un saber de los fundamentos suprafísicos de nuestras ciencias y de todo lo que hay. En ese contexto, la acuñación retórica de un metatérmino parece corresponder a toda una estrategia discursiva de fundamentación "en un orden más allá 
de lo dado" (o "en un plano superior") de las tramas de saberes, prácticas e instituciones, que habían visto erosionarse su sustento en las tradiciones mítico-religiosas de la Grecia arcaica y la cultura política de la Antigüedad. Como si, en situación de crisis de los campos discursivos, la necesidad de reencontrar un nuevo léxico de fundamentación se expresara en la articulación de "metatérminos", o significantes de un orden superior, para una reconstrucción reflexiva del saber.

De modo análogo, la acuñación de términos como metamatemática y metalenguaje nos remite a la marcada crisis de fundamentación en el terreno de las matemáticas y la lógica, que se produjo entre finales del siglo XIX y la primera mitad del siglo XX, debido a la irrupción de geometrías alternativas y en el marco de una problematización de la demostrabilidad de los fundamentos de la matemática y la lógica. Entre los años treinta y cuarenta vemos desplegarse todo un repertorio de metatérminos con una explícita connotación fundacional, como significantes de un léxico reflexivo de segundo orden o de un lenguaje de orden superior. En las obras de Hilbert vemos aparecer el término metamatemática para designar cierto lenguaje de fundamentación de la matemática. En la reflexión lógica de Russell y en su teoría de los tipos lógicos se hará presente la necesidad de distinguir el lenguaje objeto y los lenguajes de orden superior o metalenguajes. A partir de entonces los metatérminos del tipo metalenguaje no sólo tuvieron una rigurosa formalización en el ambiente logicista del neopositivismo lógico (en autores como Carnap o Tarski). Entre los años cuarenta y cincuenta se extrapolaron a otras disciplinas relacionadas con el lenguaje y la comunicación, como se puede apreciar en la acuñación de la metasemiótica y la metasemiología en la lingüística de Hjelmslev (en tanto que semióticas de segundo orden que tendrían otras semióticas como objeto); como ocurrió con la reflexión sobre la función metalingüística en la semiótica de Jakobson (esto es, la posibilidad de tematizar con el lenguaje el propio código lingüístico). O como sucedió con la apropiación del concepto de metalenguaje en la teoría de la comunicación de Bateson, y su acuñación conceptual de cierta metacomunicación (comunicación sobre el propio patrón comunicativo), que acompañaría a toda comunicación denotativa. También en el discurso filosófico y científico contemporáneo 
la articulación de metatérminos parece corresponder a cierta estrategia retórica de fundamentación discursiva, a partir de significantes marcados como de orden superior, o bien de cuestionamiento de los fundamentos disciplinares (en situación de crisis) mediante la introducción de un metadiscurso reflexivo.

Desde que, en los años setenta, Flavell acuñó los conceptos de metamemoria y metaconocimiento (probablemente por influjo del léxico del "metalenguaje" que ya circulaba en ciencias humanas), toda una serie de metatérminos se ha sumado a una nueva retórica del orden superior en psicología: metaaprendizaje, metapensamiento, metaatención, etc. (Mayor, Suengas \& González, 1995). En el caso de la psicología, esta flotación masiva de metatérminos también coincide con un escenario de crisis paradigmática en la investigación psicológica. Tras un periodo de ciencia normal (entre los años veinte y cincuenta) en que el conductismo se erigió como el paradigma hegemónico en la investigación psicológica experimental, la psicología conoció cierta revolución cognitiva, que impulsó una significativa multiplicación de programas de investigación hasta el día de hoy. No parece casual que -a partir de los ańos setenta y en el contexto de la consolidación de la epistemología genética y la psicología cognitiva de inspiración piagetiana, de la psicología del procesamiento de la información y de los enfoques socio-cognitivos- se haya producido una extraordinaria proliferación de metatérminos, que reintroducían en la investigación psicológica procesos cognitivos de orden superior (anteriormente proscritos). La incorporación de un léxico psicológico reflexivo y de segundo orden parecía prometer la superación de la situación de crisis de fundamentación paradigmática, al introducir -en los procesos cognitivos investigados- un vocabulario de términos metaprefijados, alusivos a funciones de autorregulación, así como a la capacidad de autoobservación y autocontrol.

Con el metadiscurso de los procesos metacognitivos la psicología fundamenta reflexivamente su quehacer, incorporando un psicólogo observador a escala (o una instancia de autorregulación psicológica) en cada uno de los procesos cognitivos investigados. 
Las fuentes clásicas y los paradigmas hegemónicos de la investigación sobre la metacognición

Al rastrear los orígenes del giro metacognitivo en la psicología contemporánea tenemos que remitirnos a la investigación sobre el metaconocimiento y la metamemoria en la psicología del desarrollo de inspiración piagetiana. John Flavell incorporó en sus análisis del desarrollo cognitivo los términos metaconocimiento y metamemoria, y consideró que para estudiar el desarrollo de la memoria era necesario tener en cuenta los conocimientos y procesos cognoscitivos relativos a lo que las personas saben acerca de la memoria. La metamemoria constituiría el conocimiento del cual la persona dispone acerca de sí mismas, de la tarea a realizar y de las diversas estrategias de memoria (Flavell, 1993). Este metaconocimiento de lo que las personas saben sobre sí mismas y sobre los demás como seres con memoria (la identificación de experiencias de recuerdo y olvido, o la estimación de las capacidades de la propia memoria) aporta un indicador del desarrollo cognitivo de la memoria. También el metaconocimiento de las tareas de memoria (el conocimiento del grado de dificultad y de las demandas de recuperación), así como el metaconocimiento de las estrategias de memoria (la concepción de posibles estrategias de almacenamiento y recuperación), indican aspectos cruciales del desarrollo cognitivo de la memoria.

Según Flavell, los conocimientos metacognitivos referentes a las variables de persona, tarea y estrategia, interactúan. Además, se distingue entre conocimiento metacognitivo y experiencias metacognitivas, o sea, las sensaciones que experimenta conscientemente un sujeto que está implicado en un proceso cognoscitivo (por ejemplo, darse cuenta de que la tarea que realizamos es difícil; tener la sensación de que el modo de afrontarla resulta inadecuado; o sentir que estamos cerca del objetivo de la tarea) (Flavell, 1992, 1993).

En el modelo de Flavell, la supervisión de los procesos cognoscitivos tiene lugar a través de las operaciones e interacciones entre el conocimiento metacognitivo, las experiencias metacognitivas, las metas propuestas y las estrategias disponibles (tanto las estrategias cognitivas 
que nos permiten realizar un progreso cognoscitivo, como las estrategias metacognitivas que nos permiten supervisarlo) (Flavell, 1992). Así pues, el desarrollo de la metacognición resultaría favorecido por logros evolutivos tales como la creciente conciencia de sí mismo en tanto centro de la actividad cognoscitiva, o como la cada vez mayor propositividad de nuestros procesos cognoscitivos. Además, la conciencia metacognitiva se desarrollaría con la práctica directa, y existe la posibilidad de modelarla en las escuelas (Flavell, 1987).

No sólo en la psicología del desarrollo de inspiración piagetiana circuló el concepto de metacognición. Dentro del contexto de las teorías de la cognición inspiradas en modelos de procesamiento de la información es habitual atribuir operaciones fundamentales de control ejecutivo a un procesador central, capaz de llevar a cabo una evaluación inteligente de su propio funcionamiento. Ann Brown aportó una caracterización de la metacognición en términos del control ejercido por un sistema ejecutivo central en el procesamiento de la información. Las funciones del ejecutivo central incluirían una amplia gama de tareas: la predicción de las limitaciones en la capacidad del sistema; la conciencia de las diversas rutinas heurísticas disponibles y de su dominio de utilidad propio; la identificación y caracterización del problema; la planificación de estrategias de resolución de problemas adecuadas; la supervisión de la efectividad de las estrategias aplicadas, o la determinación estratégica del tiempo de ejecución (Brown, 1987).

John Borkowski también elaboró un modelo de la metacognición basado en la psicología del procesamiento de la información y, concretamente, en la capacidad de cierto ejecutivo central para llevar a cabo la generalización y transferencia de estrategias. Borkowski desarrolló un modelo jerárquico de la inteligencia que distinguía entre un sistema arquitectónico, que garantiza la eficacia en el procesamiento y decodificación de la información y, por otra parte, un sistema ejecutivo que regula la recuperación del conocimiento, modifica la base de conocimiento y media en la resolución de problemas.

Entre los componentes básicos del sistema ejecutivo se encuentran las estrategias de control y el conocimiento metacognitivo que selecciona 
y regula las estrategias cognitivas, así como predice la generalización de estrategias. La metacognición constituye un importante factor en la transferencia de estrategias que nos permite comprender adecuadamente los fallos en la generalización de estrategias. Por tanto, si la metacognición se asocia a la propositividad, autosupervisión e inventividad, así como a procesos de control ejecutivo de orden superior, también ha de ser vinculada al mantenimiento y generalización de estrategias. La relación entre estrategias de orden superior que indican procesos de control y metacognición (las decisiones reflexivas sobre el uso o revisión de una estrategia), resultaría bidireccional: la generalización de estrategias promueve el conocimiento metacognitivo, y las habilidades metacognitivas favorecen el desarrollo de estrategias cognitivas transituacionales y de orden superior (Borkowski, 1985).

Bajo este paradigma computacional de los procesos mentales, se elaboró la versión estándar de la relación entre conciencia y control metacognitivo. Thomas Nelson y Louis Narens establecieron un marco teórico que, incluso en la más reciente investigación sobre metacognición, permite comprender la articulación del control y la conciencia metacognitivos. Esta relación entre conciencia y control metacognitivos se comprende a partir de una serie de principios: por una parte, los procesos cognoscitivos están divididos en dos o más niveles, interrelacionados como nivel-objeto y metanivel. En segundo lugar, el metanivel contiene un modelo dinámico (una simulación mental) del nivel-objeto, de modo que el sistema pueda controlar el proceso dinámico, y se representen estados finales alternativos. Por último, existen dos relaciones, llamadas control y supervisión, que se definen en términos de la dirección del flujo de información entre el metanivel y el nivel-objeto. En la relación de control, la información fluye del metanivel al nivel-objeto, de modo que se transforma el nivel-objeto o sus estados (desplegándose una acción); en la relación de supervisión, el metanivel es informado por el nivel-objeto y se transforma el modelo mental. Según Nelson y Narens, la relación de control y la relación de supervisión entre nivel-objeto y metanivel son asimétricas; se diferencian de otros modelos de relación entre nivel-objeto y metanivel que podríamos concebir (un mecanismo de control sin retroalimentación; 
un dispositivo de espionaje sin control; o un mecanismo con una relación simétrica en que ninguno de los componentes funciona como metanivel respecto del otro) (Nelson \& Narens, 1992; 1994).

Pese al impulso decisivo que recibió la investigación sobre metacognición bajo los paradigmas cognitivista y computacional, no podemos olvidar el rescate del sentido de agencia personal en los enfoques fenomenológicos de la metacognición. Desde una perspectiva fenomenológica, Bárbara McCombs ha señalado que, si bien el metaconocimiento y las destrezas metacognitivas proporcionan la estructura básica para el desarrollo del autocontrol positivo, así como para la autorregulación de los propios pensamientos y sentimientos, los agentes necesitan disponer de una autoidentidad relativamente bien definida y estable, originada en la propia autoconciencia. Los procesos metacognitivos de orden superior que controlan los procesos cognitivos y afectivo-motivacionales de orden inferior, mediante la autovaloración y autodirección, tienen como trasfondo fundamental el sentido de la agencia personal. El yo se descubre como el constructor de sus propias autoconcepciones y evaluaciones, y los procesos de control metacognitivo se constituyen como herramientas del autogobierno del agente. Con el aprendizaje de estrategias metacognitivas de autocontrol, autosupervisión y autoevaluación, se desarrolla el sentido de agencia personal; pero las disposiciones de autorregulación metacognitiva están arraigadas en la conciencia personal del yo como agente (McCombs, 1993).

Además, según McCombs, conviene distinguir entre los procesos de pensamiento metacognitivo -que operan dentro del sistema cognitivo o intelectual- $y$, por otra parte, los procesos de pensamiento de orden superior -como la creatividad o la sabiduría- que se despliegan más allá del sistema cognitivo, en el nivel de la autoconciencia del yo como agente. En los niveles más altos de autoconciencia el sistema cognitivo y los procesos intelectuales actúan como soportes de la persona, en tanto que la comprensión metacognitiva, más que constituirse como un proceso de construcción intelectual de esquemas que enmarcan y limitan el pensamiento del agente, se configura como un proceso abierto de desbordamiento y recontextualización de nuestros sistemas de creencias 
condicionados y de los marcos personales de referencia. La metacognición nos conduciría, así, a la comprensión profunda del sentido de agencia personal (McCombs, 1993).

¿Cuál es la problemática central en la actual teorización sobre la metacognición? En gran medida, la investigación contemporánea sobre la metacognición se concentra en la metamemoria, esto es, en los procesos y estructuras en virtud de los cuales las personas pueden realizar juicios, evaluaciones o comentarios tanto sobre la propia memoria como sobre el aprendizaje. Los juicios metacognitivos que mayor atención han concitado en la investigación sobre metamemoria son los juicios relativos a la sensación de saber (presentados en la literatura con las siglas FOK, esto es, feeling-ofknowing), los juicios relativos a tener algo en la punta de la lengua (juicios $T O T$, o tip-of-the-tongue) y los juicios relativos al aprendizaje (juicios JOL, o judgements-of-learning) (Metcalfe \& Dunlosky, 2008).

Los juicios relativos a la sensación de saber (FOK) nos enfrentan a la interrogante de cómo las personas que fallan a la hora de dar respuesta a requerimientos de información o a alguna tarea de reconocimiento son capaces de experimentar que sabían la respuesta, y pueden predecir correctamente que recuperarán dicha información en el futuro (aunque momentáneamente sean incapaces de recordar). La mayoría de las teorías contemporáneas no consideran que las personas tengan un acceso directo a alguna huella de memoria subliminal. Asumen que los juicios relativos a la sensación de saber se basan en alguna información parcial, ya sea debido a la familiaridad con la pregunta o con el tema de ésta; ya sea a causa de la accesibilidad parcial de información sobre el contenido de la respuesta (como la inicial de una categoría). En ese sentido, las teorías sobre la sensación de saber (o sobre el sentimiento noético) introducen algún heurístico que encamina la inferencia de la respuesta, en vez de presuponer un misterioso acceso a contenidos latentes (Metcalfe \& Dunlosky, 2008).

Uno de los tipos de juicio de sentimiento noético que más ha llamado la atención de los psicólogos (desde que William James lo describió) es aquel en que las personas dicen tener alguna información, categoría o nombre en la punta de la lengua (juicios TOT). Aunque constituyen un tipo de juicio relativo a la sensación de saber, presentan 
la especificidad de enfocarse más directamente en alguna categoría muy accesible, de modo que no implican tantas inferencias como los juicios de sentimiento noético en general. Las explicaciones teóricas de este fenómeno han enfatizado la accesibilidad a información parcial sobre el ítem buscado (como también ocurría en los juicios de sentimiento noético). Asimismo, han llamado la atención sobre la posibilidad de que frecuentemente exista una recuperación semántica que no va acompañada del acceso fonológico a la categoría buscada, e incluso han señalado la posible existencia de categorías, similares a la que intentamos recordar que bloqueen el acceso al ítem buscado (Metcalfe \& Dunlosky, 2008).

Los juicios relativos al aprendizaje (JOL) también han sido profusamente investigados como paradigma metacognitivo. Se trata del modo en que las personas evalúan qué tan bien han aprendido los contenidos requeridos, ya sea durante el curso del aprendizaje o después de haber completado la tarea de aprendizaje. En ese sentido, los juicios retrospectivos sobre el aprendizaje parecen ser más precisos que los juicios inmediatos que tienen lugar mientras se aprende. Semejante precisión se ha explicado teóricamente, apelando al hecho de que los juicios retrospectivos no se basan únicamente en información de la memoria de corto plazo (como ocurre con los juicios inmediatos), sino, también, de la memoria de largo plazo. Además, se ha observado el papel que juega el estudio adicional como clave de la mayor precisión de los juicios de aprendizaje retrospectivos; y es que, cuando el juicio se posterga, se produce cierto efecto de profecía de autocumplimiento que conduce a reforzar el estudio de aquellos contenidos cuyo aprendizaje se estima bajo. A esto habría que añadir el rol que juegan, en tanto factores clave para los juicios de aprendizaje, la familiaridad con las pistas, la fluidez en el procesamiento de las categorías durante el estudio, el recuerdo del resultado de pruebas anteriores, entre otros (Metcalfe \& Dunlosky, 2008).

Cabe preguntarse qué función podrían desempeñar estos juicios metacognitivos, dado que siempre es factible que se produzcan ilusiones metacognitivas, e incluso puede ocurrir que las personas no conviertan este metaconocimiento en estrategias de control idóneas. Por eso, la investigación reciente parece más interesada en explicitar qué 
formas de control del aprendizaje y de la memoria se siguen de nuestros juicios metacognitivos. Los juicios de sentimiento noético cumplen un importante rol motivacional: guían la toma de decisiones y hacen que las personas perseveren en la búsqueda de respuestas (o desistan del intento de recuperación, en ausencia del sentimiento noético). De modo análogo, los juicios relativos al aprendizaje determinan decisiones idóneas acerca de qué es lo que se va a estudiar, cómo se va a distribuir el aprendizaje y cuánto se persistirá en la tarea; contribuyen a un aprendizaje más efectivo (Metcalfe \& Dunlosky, 2008).

Algunos problemas epistemológicos implicados por la metacognición

¿De qué modo es posible obtener un conocimiento de qué conocemos y de cómo lo sabemos? ¿Qué tipo de conocimiento es ese metaconocimiento? Las interrogantes epistemológicas que rodean a la metacognición son tan sofisticadas como la propia idea de un metaconocimiento y un metacontrol de nuestros procesos psíquicos.

El primer problema que plantea la metacognición se refiere a si -al plantear la existencia de una supervisión y control internos de nuestra actividad cognitiva - no estaremos reintroduciendo al fatídico "homúnculo" (ese parche explicativo consistente en apelar a un hombrecillo o personita interior, para dar cuenta de toda nuestra actividad), que ha sido tan denostado en la filosofía de la mente y en la neurociencia contemporáneas. No parece muy razonable dar cuenta de nuestra actividad cognitiva y conductual, atribuyéndosela a un misterioso "fantasma en la máquina", esto es, a una intangible entidad personificada y separada del cuerpo. Al tratar de dar cuenta de nuestra actividad mediante el homúnculo, tan sólo estamos duplicando aquello que había que explicar (ahora, tendríamos que explicar no sólo nuestra actividad sino, también, el modo de proceder del homúnculo). Además, la apelación al homúnculo constituye un manifiesto error categorial, ya que el complejo entramado de nuestra actividad cognitiva y conductual es reducido a una cosa localizable dentro del cuerpo (Ryle, 2005). 
¿Incurre la teorización de la metacognición en la falacia del homúnculo? En aquellos modelos de la metacognición que privilegian el acceso de primera persona, enfatizando el autogobierno del yo y el sentido de agencia personal, puede parecer obvia la tentación de personificar-en un homúnculo interno- las condiciones de posibilidad de la metacognición. No obstante, una descripción fenomenológica rigurosa de los modos de hacer sentido y de los horizontes intencionales, tanto de nuestra actividad consciente como del sentido de agencia, excluye semejante cosificación del quehacer subjetivo. Paradójicamente, en los modelos de metacognición basados en la psicología del procesamiento de la información (y en gran medida deudores de una filosofía de la mente funcionalista que -para eliminar cualquier homúnculo- reducen la actividad consciente a procesos de computación simbólica, o sea de almacenamiento y transformación regulada de información entrante, para ejecutar cierta respuesta), la atribución de las operaciones complejas de control ejecutivo a una instancia supervisora interior al sistema genera el problema teórico de quién o qué realiza el control ejecutivo. En este punto, topamos con la paradójica afirmación de un homúnculo interior al sistema que se plantea como explicación, en última instancia, de aquellos procesos que el mecanismo de procesamiento de información pretendía explicar (Searle, 1996). En cualquier caso, la investigación contemporánea sobre metacognición parece tener una clara conciencia de los riesgos de cosificar el autocontrol y supervisión cognitivos en algún homúnculo o en algún módulo ejecutivo especial con características homunculares (Rosenthal, 2000; Metcalfe, 2008).

Una segunda dificultad suscitada por la metacognición consiste en la llamada paradoja de Comte, esto es, en los argumentos con que dicho autor impugnaba la introspección psicológica: ¿cómo es posible que la conciencia pueda desarrollar su actividad y, al mismo tiempo, observar la actividad que desarrolla? (Metcalfe, 2008). ¿Queda atrapada en estas paradojas la investigación sobre metacognición? En principio, no, ya que la formulación de esta paradoja de autorreferencia cognitiva presupone una ilusoria conciencia unitaria e indivisible, como si el sujeto cognoscente y el objeto conocido fueran una misma entidad. Pero semejante visión 
de una conciencia unitaria e indivisible ha resultado reiteradamente impugnada por la neurociencia contemporánea, que nos ha familiarizado con modalidades de procesamiento en paralelo y con formas de retroalimentación y monitoreo en múltiples niveles, sin necesidad de un supervisor central único. Además, la actual investigación en metacognición suele aceptar el modelo de Nelson y Narens que establece dos niveles de conciencia (un nivel-objeto y un metanivel), en vez de presuponer una conciencia homogénea y unitaria (Metcalfe, 2008).

Otra interrogante epistemológica que suscita la metacognición concierne a si ésta implica una remisión al infinito, de tal modo que cada nivel de metaconocimiento o metacontrol precise de otro de orden superior, y así, ad infinitum. Esta paradoja se disuelve tan pronto como reconocemos que no es preciso remitir a un supervisor de orden superior (jerárquicamente privilegiado y distanciado) toda actividad de monitoreo o de control. La actual neurociencia ha aportado descripciones de la actividad mental que hacen innecesaria una jerarquía de observadores incrustados. Así se desprende de las concepciones de la actividad mental como procesos encarnados; pero, también, se sigue de las teorías que reconocen la modularidad de los procesos mentales o investigan las formas de procesamiento distribuidas en paralelo (Metcalfe, 2008). Además, dado que frecuentemente nuestro acceso metacognitivo a los estados mentales no es consciente (y, con frecuencia, no nos damos cuenta de realizar procesos metacognitivos), no hay por qué asumir que la conciencia metacognitiva genera un regreso al infinito en una jerarquía metacognitiva ilimitada (Rosenthal, 2000).

La última interrogante relativa a la metacognición nos va a permitir abordar las modalidades de conocimiento que se asocian a la metacognición: ¿es el conocimiento metacognitivo autoevidente? Así parecen asumirlo algunas concepciones de la metacognición que afirman la posibilidad de obtener un acceso vívido a las impresiones de nuestros contenidos cognoscitivos y un monitoreo directo de las huellas de nuestra memoria, a partir de los cuales formularíamos nuestros juicios referentes a la sensación de saber. Desde ese punto de vista, los juicios metacognitivos expresarían una intuición noética autoevidente, y la experiencia subjetiva 
controlaría nuestras acciones. Sin embargo, la actual investigación sobre metacognición ha insistido en el carácter heurístico de nuestros juicios metacognitivos: éstos se basan en procesos inferenciales (a partir de la familiaridad con ciertas pistas o de la accesibilidad de información parcial), más que en alguna intuición noética autoevidente. Además, nuestros juicios metacognitivos pueden ser errados $y$, como sucede en estados conscientes confabulados, nuestra sensación subjetiva de saber puede diferir marcadamente del contenido cognitivo al que se refieren nuestros juicios metacognitivos (Rosenthal, 2000). Parece más sensato reconocer que nuestros juicios metacognitivos y sentimientos noéticos son un resultado -más que la causa única- de los procesos, del desempeño y del logro, asociados a nuestras tareas cognitivas (Koriat, 2000).

Tal vez, en la teorización de las formas de metaconocimiento y metacontrol haya que renunciar a toda ilusión de transparencia y de control plenos. Al menos así se desprende de las conclusiones a que ha llegado la actual investigación sobre metacognición, cuando se asume que los procesos metacognitivos conciernen tanto a procesos implícitos y automáticos, como a procesos explícitos y controlados. No en vano los juicios metacognitivos tienen una doble faz: se basan en inferencias heurísticas implícitas y automáticas que inducen un sentimiento noético explícito, capaz de controlar nuestras acciones. Asimismo, en nuestros juicios metacognitivos hay que distinguir aquellos juicios basados en alguna información, y que involucran procesos cognitivos más analíticos, conscientes y controlados, y por otra parte, los juicios basados en la experiencia (como ocurre en los juicios TOT), que se asocian a ciertos sentimientos o sensaciones subjetivos (Koriat, 2000). La gama de juicios metacognitivos va desde la expresión de la sensación de saber, hasta la formulación reflexiva de juicios noéticos, e implica tanto procesos automáticos y tácitos, como operaciones explícitas y conscientes. Sea como sea, el metaconocimiento no garantiza una conciencia transparente y autoevidente de toda nuestra actividad cognitiva. Tampoco implica un control exhaustivo de nuestra actividad, ya que el darse cuenta subjetivamente suele ser posterior a la toma de decisiones y al control de la conducta (Rosenthal, 2000). 
Además de los problemas epistemológicos mencionados, existe toda una problemática teórica asociada a las perspectivas epistemológicas en la atribución de metaconocimiento. Ya que la metacognición resulta caracterizada como una forma de conocimiento conducente a la formulación de juicios, cabe preguntarse desde qué perspectiva puede llevarse a cabo la atribución de metaconocimiento. ¿¿Se puede atribuir metaconocimiento a un sistema psíquico a partir de condiciones objetivables? ¿La atribución de metaconocimiento requiere de una autoatribución basada en la experiencia vivencial y el sentido de agencia personales? Este tipo de interrogantes introducen un dilema que, tal vez, haya que superar: ¿es el metaconocimiento público o privado?

En gran parte de la investigación psicológica sobre metacognición (y congruentemente con la metodología científica convencional) ha predominado una perspectiva de "tercera persona", centrada en la descripción externa de condiciones objetivables y de mecanismos estructuralmente determinados que hacen posible el metaconocimiento. En los modelos de la metacognición basados en el procesamiento de la información, el metaconocimiento se perfila como un conjunto de dispositivos de monitoreo y control (como modalidades de computación: ya sea el procesamiento secuencial de procesos en paralelo, el incrustamiento recursivo de las representaciones, o bien, la operación de heurísticos inferenciales); los procesos se objetivan como un conjunto de fenómenos estructuralmente determinados accesibles a cualquier observador. Ahora bien, la atribución de metaconocimiento en tercera persona -tal como ocurre en la psicología del procesamiento de información- corre el riesgo de omitir la intencionalidad intrínseca de los procesos metacognitivos (esto es, la apertura y capacidad de autotrascendencia, la iniciativa subjetiva y el sentido de agencia), asimilándola a la capacidad de transformación computacional de información para generar respuestas programadas (Searle, 1996).

Algunas teorías sobre la metacognición han apostado por rescatar el sentido de agencia personal, o las formas de autoconciencia y autotrascendencia del yo, como presupuesto indispensable para que el metaconocimiento cobre sentido. Es el caso de las aproximaciones fenomenológicas 
a la metacognición (McCombs, 1993). En este tipo de descripciones, que articulan la experiencia vivencial del sujeto metacognitivo, puede decirse que se adopta una perspectiva de primera persona. La atribución de metaconocimiento en primera persona se da, pues, como comprensión vivencial e intencional de los horizontes de sentido de nuestra actividad cognitiva que puede ser descrita fenomenológicamente desde la intimidad e inmediatez de nuestra experiencia cognitiva y nuestro propio sentido de agencia.

¿Estamos forzados a elegir entre las alternativas de una atribución de metaconocimiento en primera persona o en tercera persona, entre un acceso privado y otro público? La atribución de metaconocimiento (el reconocimiento de que estamos en presencia de juicios metacognitivos) requiere de marcos interpretativos y de un trasfondo de comprensión compartidos que nos permiten asignar un significado cabal a la expresión de creencias metacognitivas. Asimismo, la autoatribución de metaconocimiento no consiste en una inefable intuición noética; se articula de acuerdo a pautas de significado y patrones de desempeño intersubjetivamente compartidos: la descripción privada está siempre públicamente estructurada. Cabe hablar, entonces, de una perspectiva de segunda persona: lo que aparece como comportamiento externo puede considerarse como huella o expresión de la actividad mental de un agente humano racional, poseedor de creencias, deseos y estados intencionales. Desde esta perspectiva, la interpretación intencional de la actividad cognitiva de otro agente permitiría reconocer los modos intencionales en que ese otro supervisa y controla metacognitivamente sus procesos cognitivos. Esta perspectiva de segunda persona se podría caracterizar como "heterofenomenológica": aporta un relato neutral y comprensivo de cómo se lleva a cabo la actividad intencional en otro sujeto, en términos intersubjetivos, y da la mejor interpretación que podamos elaborar (Dennett, 1995).

Semejante perspectiva intersubjetiva (ponerse en el lugar del otro, en virtud de los patrones de significado y de desempeño compartidos) se encuentra en aquellos enfoques sociocognitivos sobre la metacognición, que han insistido en que la autorregulación psíquica es el resultado de la 
internalización de procesos que en su inicio eran socialmente compartidos. En ese sentido, la investigación de inspiración vygotskiana asume que, gracias a la mediación del habla interna, las funciones aparecen dos veces en el desarrollo psíquico: primero entre personas, como una categoría interpsicológica, y luego, dentro del psiquismo del individuo, como una categoría intrapsicológica. Ahora bien, el paso de la regulación externa a la regulación interna no constituiría una transposición pasiva y simple. Sólo si existe en el individuo una verdadera reconstrucción y transformación activa de los procesos interactivos podemos hablar de un componente metacognitivo en la heterorregulación. En definitiva, la autorregulación psíquica se podría interpretar como el resultado de la internalización de cierto diálogo primordial heterorregulatorio (Wertsch, 1991).

No hay por qué forzar una elección unilateral de alguno de estos enfoques de la actividad metacognitiva. El metaconocimiento (lo mismo que el conocimiento humano) se sustenta simultáneamente en esas tres perspectivas: la del autoconocimiento, la del conocimiento objetivador (del modo en que funcionan nuestros procesos cognitivos) y la del conocimiento intersubjetivo. También en el caso de la metacognición, los tres tipos de conocimiento forman un trípode, y si se perdiera alguna de las patas no se sostendría la posibilidad del metaconocimiento. Como Davidson plantea, sólo puedo calibrar mis pensamientos a través de una segunda persona, y puedo calibrar lo que piensan los demás al saber lo que yo pienso; además, puedo calibrar el pensamiento ajeno (y el propio) porque comparto un mundo con otros y reacciono del mismo modo ante el mundo (Davidson, 2003: 299-300). En la construcción de nuestra experiencia metacognitiva jugamos constantemente con estas perspectivas: accedemos vivencialmente a nuestra conciencia intencional, tanto como nos ponemos en el lugar del otro sujeto a partir de un trasfondo de sentido compartido, o como tratamos de objetivar públicamente las condiciones de los procesos cognitivos y metacognitivos. Varela entendió muy bien que nos encontramos ante un continuo de perspectivas complementarias en la atribución de conciencia, que va desde la intimidad de la vivencia personal, pasando por la mediación del otro, a la validación pública de los procesos cognitivos (Varela, 2000: 306). 
La construcción social de la metacognición

Las condiciones epistemológicas de inscripción y circulación de un concepto científico o un enfoque teórico no son separables de los contextos sociohistóricos de su irrupción; existe una relación sumamente permeable entre los factores intracientíficos y los aspectos aparentemente extracientíficos que conducen a la acuñación teórico-conceptual.

En la reconstrucción genealógica del surgimiento de las ciencias humanas se ha insistido en la complicidad que existe entre el nacimiento de disciplinas como la psicología y una nueva configuración de los procedimientos de individualización, a través de los aparatos disciplinarios de la sociedad moderna. En la formación social moderna, el examen constante, la documentación científica de la diferencia individual en tanto caso, así como la comparación con la norma y la normalización consiguiente (o sea, los procedimientos de análisis y aplicación de ciencias humanas como la psicología), se han convertido en los dispositivos centrales de toda una tecnología de poder disciplinaria (como medios de movilización de los cuerpos, o mecanismo de administración de sus fuerzas y aptitudes). El examen y la normalización psicológica operarían como un dispositivo científico-disciplinario de individualización, que constituye al individuo en tanto efecto y objeto de un nuevo saber-poder, ejercido reticular y cotidianamente en los ámbitos nucleares de concentración disciplinaria de la sociedad civil (la escuela, la fábrica, la clínica o la prisión) (Foucault, 1986: 175-198).

Del mismo modo que el resto de las ciencias humanas que, al alero de la ideología positivista de la ciencia, se concibieron como instrumentos de reforma y control social, la primera oleada de investigación psicológica se proyectó básicamente como una intervención psicotécnica, una herramienta de análisis de la psicología individual y un instrumento de normalización social. A este proyecto responde el desarrollo de los tests mentales para determinar (y distribuir) las diferencias individuales, en el contexto de las nuevas formas de movilización disciplinaria de la fuerza de trabajo, basadas en la escolarización universal y obligatoria. Esta iniciativa psicométrica se exportaría (con el mismo propósito de homogeneización y distribución 
de las diferencias individuales) a otras instituciones de concentración disciplinaria tan decisivas como la escuela, los cuarteles y fábricas, o esos ámbitos de encierro disciplinario intensivo que son las prisiones. También los inicios de la psicopatología participan de esta vocación psicotécnica de aplicar el examen de los casos individuales (en la institución total de la clínica), al servicio de la normalización social (Leahey, 2005: cap. 6). Entre el nacimiento de la investigación científico-disciplinaria de la psicología, a finales del Siglo XIX y comienzos del siglo XX, la psicología operó como una tecnología de la normalización individual y asumió como ámbito de estudio la psicología de la conciencia individual (ya fuese bajo métodos de introspección experimental o métodos fenomenológicos). Se trata de un proyecto científico-disciplinario funcional a las exigencias de movilización, normalización y homogeneización de la fuerza de trabajo, en un periodo de franca expansión del capital (que implicó la exigencia de una distribución y control exhaustivos de las fuerzas productivas, incluida la reproducción de sujetos estandarizados).

Entre los años veinte y cincuenta el proyecto de investigación de la psicología cambió de curso, en consonancia con las nuevas exigencias de movilización y control social de la fuerza laboral. El primado del conductismo como paradigma hegemónico de la psicología experimental revela un desplazamiento del foco de interés, desde la normalización individual en entornos de concentración disciplinaria, hasta la ingeniería del comportamiento y el control de la conducta social a gran escala (ejercido por medio de la planificación experimental de ambientes, y con el propósito de seleccionar conductas adaptativas). Semejante mecanicismo adaptativo, que popularizó cierto modelo del ser humano como autómata reactivo (y de la psicología, como tecnología conductual) (Leahey, 2005: cap. 11), corresponde a los modelos maquínicos de movilización industrial que son propios de la fase fordista del capitalismo desarrollado. Bajo el fordismo, la organización de los procesos productivos (cada vez más sujetos a una concentración monopolística del capital y a la intervención del Estado como actor económico) demanda una creciente mecanización y automatización, una adaptabilidad de la fuerza laboral a las exigencias de un trabajo cada vez más abstracto y estandarizado, así como una unificación progresiva de la 
producción y del consumo. Consecuentemente, la ingeniería conductista del comportamiento acometió una empresa de control experimental de la conducta, de condicionamiento conductual a ambientes genéricos y de integración de secuencias de estímulos y respuestas adaptativos, que se aplicó en el diseño de ambientes de condicionamiento en escuelas y fábricas. El énfasis había pasado del examen y normalización de la psicología individual, al control y planificación de ambientes genéricos de condicionamiento.

¿A qué condiciones sociohistóricas de la movilización productiva corresponde el giro metacognitivo de la psicología contemporánea? Desde los años setenta se han puesto en el centro de la investigación las capacidades de monitoreo y control que hacen posibles un aprendizaje autorregulado y procesos cognitivos de orden superior. Este énfasis en la autorregulación y control reflexivo no es casual; responde a una marcada inquietud social ante la crisis en los sistemas educativos, toda vez que éstos ya no pueden hacerse cargo de las nuevas exigencias que la producción social dirige a la escuela. Desde los setenta se ha visto en la investigación y desarrollo tanto de la metacognición como del aprendizaje autorregulado, la clave para enfrentar la crisis de los sistemas educativos: se generalizaría la capacidad de aprender a aprender, y se acabaría con la mecánica reproducción y atesoramiento de conocimiento inerte.

Todo este proyecto científico-educativo (psico-pedagógico) de desarrollo metacognitivo -así como la crisis de los sistemas educativos a que éste responde- arraiga en las nuevas exigencias de movilización y organización social, en la etapa posfordista del modo de producción capitalista, al alero de la crisis del Estado de bienestar desde los años setenta. El hecho de que las capacidades cognitivas sean una fuerza productiva central en una economía postindustrial (terciarizada e informacional) genera un desfase entre los roles tradicionales de la escuela (disciplinarios o de asimilación de conocimiento genérico) y los nuevos requisitos de la producción social. No en vano, la fase posfordista del capitalismo desarrollado se caracteriza por la alta movilidad y desmaterialización de los flujos productivos, por la informatización general de la sociedad, por la movilización del intelecto y las capacidades cognitivas como recursos 
productivos, por el crecimiento exponencial del conocimiento (en lo que se ha llamado la "sociedad del conocimiento") y por la apropiación del capital intelectual. El giro metacognitivo también parece corresponder al carácter fluido y heurístico (metamnemónico) de las nuevas tecnologías de la información y comunicación, que el posfordismo moviliza masivamente. Éstas -a diferencia de la inscripción literariafluidifican y operacionalizan la memoria cultural hasta tal punto, que el recuerdo queda ligado a la movilización de procedimientos estratégicos de búsqueda, gestión y almacenamiento de la información y el conocimiento. Ese despliegue reticular de facultades metamnemónicas y metacomunicativas (disposiciones relacionales, capacidades reflexivas y estrategias procedimentales) resulta funcional a la deriva posfordista del capitalismo contemporáneo. Desde ese punto de vista, el proyecto psicopedagógico implícito en el giro metacognitivo de la psicología responde a la crisis de legitimidad de las formas de control social de gran escala (sobre todo de aquellas que se despliegan en la escuela tradicional), promoviendo una nueva modalidad de gestión de las capacidades cognitivas, tan autónoma y reflexiva, como descentrada, fluida y operacional. La psicología metacognitiva constituye la forma psicopedagógica y reflexiva de autorregulación y autocontrol en la organización social postfordista.

\section{Conclusión}

A través de nuestra investigación genealógica y epistemológica se ha podido apreciar que la dispersión de las acepciones del concepto de metacognición responde a la propia heterogeneidad de los paradigmas psicológicos desde los que fue construido este término. No sólo se observan formas de inconmensurabilidad entre los presupuestos epistemológicos subyacentes a los distintos paradigmas en liza que contribuyeron inicialmente a la acuñación del concepto (sobre todo en lo relativo al lugar de la conciencia y del sentido de agencia). Además, cabe reconocer un proceso de normalización de la investigación sobre metacognición, en el 
que las disputas sobre paradigmas han ido dando paso a una articulación mesoteórica de modelos aceptables y de diseños experimentales enfocados en fenómenos metacognitivos específicos.

En este mismo proceso han menguado las expectativas acerca de la posibilidad de una conciencia reflexiva de la actividad cognoscitiva y sobre las opciones de utilizar ese autoconocimiento para un control metacognitivo exhaustivo. Al acotarse la construcción experimental de los fenómenos metacognitivos, las especulaciones acerca de una conciencia transparente y de un control pleno han perdido peso: la reciente investigación en metacognición reconoce que los procesos metacognitivos se asocian a inferencias heurísticas, abarcan operaciones automáticas y tácitas -tanto como la reflexión explícita y consciente- y, además, involucran desde la sensación de saber hasta la formación de juicios noéticos. De ese modo, la investigación en metacognición se ha desmarcado de las perspectivas epistemológicas de primera persona, basadas en la autoevidencia subjetiva. Semejante perspectiva subjetiva se ha integrado con los enfoques de tercera persona, que posibilitan la objetivación de las condiciones de la metacognición, e incluso con los enfoques de segunda persona, que asumen el rol que juega la atribución interpersonal de metaconocimiento, esto es, el sentido social de la metacognición.

A propósito del aspecto social de la metacognición, nuestra investigación ha podido reconstruir las operaciones discursivas y el trasfondo sociohistórico, a partir de los cuales se inscribe y construye el sentido de la metacognición. No sólo hemos asociado la acuñación del vocabulario metacognitivo a la flotación de un metadiscurso de autofundamentación, que expresa una perspectiva epistemológica reflexiva y de segundo orden en el léxico psicológico contemporáneo. El sentido crítico de nuestra investigación nos ha permitido patentizar la particular imbricación epistemológica de conocimiento e interés, que subyace a la acuñación conceptual de la metacognición; hemos podido apreciar que el giro metacognitivo corresponde a las nuevas modalidades de gestión de las capacidades cognitivas, en la actual etapa de la organización social de la producción. 


\section{Referencias}

Borkowski, J. G. (1985). Signs of intelligence: strategy generalization and metacognition. En S. Yussen (Ed.). The growth of reflection in children (pp. 105-144). Orlando: Academic Press.

Brown, A. L. (1987). Metacognition, executive control, self-regulation, and other more mysterious mechanisms. En F. Weinert y R. Kluwe (Eds.). Metacognition, motivation and understanding (pp. 65-116). Hillsdale: LEA.

Davidson, D. (2003). Subjetivo, intersubjetivo, objetivo. Madrid: Cátedra.

Dennett, D. (1995). La conciencia explicada. Barcelona: Paidós.

Flavell, J. H. (1987). Speculations about the nature and development of metacognition. En F. Weinert \& R. Kluwe (Eds.). Metacognition, motivation and understanding (pp. 21-29). Hillsdale: LEA.

Flavell, J. H. (1992). Metacognition and cognitive monitoring. En T. O. Nelson (Ed.). Metacognition: Core readings (pp. 3-8). Boston: Allyn \& Bacon.

Flavell, J.H. (1993). El desarrollo cognitivo. Madrid: Visor.

Foucault, M. (1986). Vigilar y castigar. Madrid: Siglo XXI.

Hofer, B. K. \& Sinatra, G. M. (2010). Epistemology, metacognition and self-regulation: musings on an emerging field. Metacognition and Learning, 5, 113-120.

Koriat, A. (2000). The feeling of knowing: some metatheoretical implications for consciousness and control. Consciounesss and Cognition, 9(2), 149-171.

Leahey, T. H. (2005). Historia de la Psicología (6a ed.). Madrid: Pearson.

Martí, E. (1995). Metacognición: Entre la fascinación y el desencanto. Infancia y Aprendizaje, 72, 9-32.

Mayor, J., Suengas, A. \& González, J. (1995). Estrategias metacognitivas. Madrid: Síntesis.

McCombs, B. L. (1993). Intervenciones educativas para potenciar la metacognición y el aprendizaje autorregulado. En J. Beltrán, V. Bermejo, M. D. Prieto \& D. Vence(Eds.). Intervención psicopedagógica (pp. 221-229). Madrid: Pirámide.

Metcalfe, J. (2008). Evolution of metacognition. En J. Dunlosky \& R. Bjork (Eds.). Handbook of Metamemory and Memory (pp. 29-46). New York: Psychology Press.

Metcalfe, J. \& Dunlosky, J. (2008). Metamemory. En H.L. Roediger (Ed.) Learning and Memory: A Comprehensive Reference (pp. 349-362). Oxford: Elsevier. 
Nelson, T. O. \& Narens, L. (1992). Metamemory: A theoretical framework and new findings. En T. O. Nelson (Ed.). Metacognition: Core readings (pp. 117-130). Boston: Allyn \& Bacon.

Nelson, T. O. \& Narens, L. (1994). Why investigate metacognition? En J. Metcalfe \& A. P. Shimamura (Eds.). Metacognition (pp. 1-25). Massachusetts: MIT Press.

Parker, I. \& Burman, E. (2008). Critical psychology: four theses and seven misconceptions. Hellenic Journal of Psychology, 5, 99-115.

Rosenthal, D. M. (2000). Consciousness, content, and metacognitive judgments. Consciousness and Cognition, 9 (2), 203-214.

Ryle, G. (2005). El concepto de lo mental. Barcelona: Paidós.

Searle, J. R. (1996). El redescubrimiento de la mente. Barcelona: Grijalbo.

Varela, F. (2000). El fenómeno de la vida. Santiago de Chile: Dolmen.

Wertsch, J. V. (1991). Voces de la Mente. Madrid: Visor.

Fecha de recepción: 20 de abril de 2010.

Fecha de aceptación: 05 de julio de 2010. 